\title{
Relation between Solar Wind Parameter and Geomagnetic Storm Condition during Cycle-23
}

\author{
Balveer S. Rathore*, Dinesh C. Gupta, K. K. Parashar \\ School of Study in Physics, Jiwaji University, Gwalior, India \\ Email: ${ }^{*}$ balveer singhra@yahoo.co.in
}

Received 22 September 2014; revised 20 October 2014; accepted 15 November 2014

Copyright (C) 2014 by authors and Scientific Research Publishing Inc.

This work is licensed under the Creative Commons Attribution International License (CC BY). http://creativecommons.org/licenses/by/4.0/

(c) (i) Open Access

\begin{abstract}
In the present paper dependence of geomagnetic activity on the solar-wind plasma and interplanetary magnetic field (IMF) parameters has been studied. We have taken interplanetary solar wind data at the instant of Dst minimum. Our study consists of 200 geomagnetic storms weighed by disturbance storm time (Dst) $<-50 \mathrm{nT}$, observed during solar cycle 23 . The study suggests that the strength of the geomagnetic storm is strongly dependent on the total magnetic field $B_{\text {total. }}$ The correlation $(\mathbf{- 0 . 7 2 )}$ has been found reasonable. In perspective of previous studies, the strength of the geomagnetic storm is strongly dependent on the southward component $(\mathrm{Bz})$ whereas in present study exposes that the correlation (0.22) is weak. This result indicates that solar wind southward magnetic field component $\mathrm{Bz}$ has significant growth particularly before the main phase of geomagnetic storm (not during the main phase). The present result implies that neither density nor temperature is significantly related to the variation of geomagnetic disturbance; rather the effects of the pressure and speed. However, a low plasma beta during highly geoeffective event seems to be an important criterion.
\end{abstract}

\section{Keywords}

Geomagnetic Storm, Interplanetary Magnetic Field (IMF), Disturbance Storm Time (Dst), Solar Cycle

\section{Introduction}

It is well-known that a continuous flow of plasma which coming out of the sun is known as solar wind. Because

"Corresponding author.

How to cite this paper: Rathore, B.S., Gupta, D.C. and Parashar, K.K. (2014) Relation between Solar Wind Parameter and Geomagnetic Storm Condition during Cycle-23. International Journal of Geosciences, 5, 1602-1608. 
of the solar wind, the Earth is heated by the hot, magnetized, supersonic collision less plasma carrying a large amount of kinetic and electrical energy. Some of this energy finds its way into our magnetosphere creating turmoil in geomagnetic activity resulting into geomagnetic storms, substorms as well as aurora [1]. It has been investigated the yearly occurrences of geomagnetic storms are not a mirror reflection of yearly variation of sunspots, but yearly occurrence of geomagnetic storms exactly follows the yearly occurrence of Halo CMEs [2].

The occurrence of geomagnetic storms is well associated with Earth-directed coronal mass ejections (CMEs), which appear in coronagraph images as bright halos around the occulting disk. CMEs are eruptions of the solar magnetic field and plasma into interplanetary space, which occur following a large-scale magnetic rearrangement in the solar atmosphere (Cremades et al., 2006). Geomagnetic storms depends upon the orientation of the magnetic field in CME; the Earth-directed CME may or may not have an intense southward Bz field. Hence, the origin of CME, the structure of their source regions and their signatures in the solar wind near the Earth, are the fundamental interest in the physics of the Sun, space plasma and space weather research. When CME enters into the interplanetary medium it is known as ICME and this ICME produced interplanetary shock (IP shock) in flowing plasma. Magnetic field frozen into plasma coming out from sun is called interplanetary magnetic field (IMF) in interplanetary medium. The southward field of IMF causes magnetic reconnection of the dayside magnetopause, rapidly injecting energetic particles into the Earth's nightside magnetosphere, which are also subjected to forces due to the magnetic field curvature and gradient as well as forces due to gyration effects. For charges of the same sign these forces act in unison, with the net effect of the protons drifting from midnight toward dusk and the electron drifting from midnight toward dawn. This oppositely directed drift comprises a ring of current around the Earth [3]. An enhanced ring current is the prime indicator of a magnetic storm. The initial feature of a geomagnetic disturbance is a sudden increase in the horizontal component of the geomagnetic field $\mathrm{H}$ observed in many stations. The geomagnetic index Dst is used to monitor the worldwide magnetic storm level. It is constructed by averaging $\mathrm{H}$ from mid-latitude and equatorial magnetograms from all over the world. Negative Dst values indicate that a magnetic storm is in progress, and the more the negative Dst the more the intensity of the magnetic storm is. These negative deflections in the Dst are caused by the ring current intensification, which flows around the Earth from east to west in the equatorial plane.

Today's challenge for Space Weather research is to quantitatively predict the dynamics of the magnetosphere and ionosphere from measured solar wind interplanetary magnetic field conditions. A number of correlative studies between the geomagnetic storm and the various interplanetary field/plasma parameters have been performed in the past to search for causes of geomagnetic activity and for developing models for predicting the occurrence of GMSs [4]-[8].

\section{Selection Criteria and Data Analysis}

In the previous work researcher are used different indices like Kp, ap and $A_{E}$. In present study, we used Dst (Disturb Storm Time) indices, that record the number and severity of geomagnetic storms during a solar cycle23. Dst index (Sugiura, 1964) [9] is defined as the hourly average of the deviation of H (horizontal) component of magnetic field measured by several ground stations in mid to low latitudes and represents the degree of equatorial magnetic field deviation specifying the magnitude of GMSs. Hourly Dst indices were obtained from the World Data Center at the University of Kyoto database. During study period we are observed 200 geomagnetic storm days with criteria Dst $\leq-50 \mathrm{nT}$.

Interplanetary magnetic field (IMF) or solar wind plasma data with $1 \mathrm{~h}$ time resolution were obtained from the OMNI website. OMNI data centre provide magnitude of average field vector also known as total magnetic field $\mathrm{B}_{\text {total }}$ or B(nT) of IMF. Negative Z-component of IMF Bz (nT) and electric field E (mV/m). They also provide proton temperature $\mathrm{T}(\mathrm{K})$, Proton density, plasma speed $\mathrm{V}(\mathrm{km} / \mathrm{s})$, flow pressure $\mathrm{P}(\mathrm{nPa})$ and plasma beta.

\section{Result and Discussion}

In present study, minimum value (maximum depression) of the Dst has been considered as storm indicator, which are further correlated with the various parameters of solar wind at the time of Dst minimum. The solar wind plasma and field measurements with $1 \mathrm{~h}$ time resolution were obtained from the OMNI website. The number of storms for Dst $\leq-50 \mathrm{nT}$ have been recorded 200 over the time period 1996-2007. The correlation coefficients ( $\mathrm{r}$ ) for all the seven individual parameter with the Dst ( $\mathrm{B}_{\text {total }}$ vs Dst, Bz vs Dst, density vs Dst, V vs Dst, $\mathrm{T}$ 
vs Dst, plasma beta vs Dst, and pressure vs Dst) have been calculated. These values are depicted in Figures 1-7.

When solar wind flows along the interplanetary medium, there is a possibility of interactions with interplanetary magnetic field structures and solar wind might be controlled by the total magnetic field ( $\left.\mathrm{B}_{\text {total }}\right)$. The Figure 1 exposes that the correlation between $B_{\text {total }}$ and Dst is as strong as -0.72 , implying that the strength of the geomagnetic storm is strongly dependent on the total magnetic field at the instant of Dst minimum. Therefore, the mechanism might be: the stronger the solar wind and interplanetary magnetic field the stronger geomagnetic storm is.

In Z-component of IMF, the orientation of interplanetary magnetic field (IMF) will be either southward or northward. The component of IMF denoted by negative sign is the southward polarity while the pressure is raised due to the coronal mass ejections (CMEs), and a geomagnetic storm is generally noticed, earlier workers suggested that for the intense storms, the larger negative Bz gives the stronger negative Dst [4] [10]-[15]. Therefore, the stronger the southward polarity field orientation, the more the depression in geomagnetic disturbance storm time is generally expected.

Figure 2 shows Dst and corresponding value of Z-component of interplanetary magnetic field, Bz at the instant of Dst minimum. When magnetic storms gain its peak value, which is called main phase of the storm. Figure 2 shows the plot of Dst against the corresponding values of Bz component of IMF. It is noted that in the previously reported work, researcher only focused on southward turning of Bz, but in present work, we have taken both southward and northward orientation of Bz. In the figures scatter point are large and large Bz values are associated with wide range of Dst values in figure. This indicates two possibilities, there may be some relationship between Dst and southward direction of $\mathrm{Bz}$ or may be some relation between Dst and northward directed Bz. Southward and northward turning of Bz with Dst are shown in Figure 2. The correlation has been calculated, the highest correlation in the range Dst $\leq-50 \mathrm{nT}$ is 0.22 . According to previous studied the strength of the geomagnetic storm is strongly depend on the southward component Bz. But in present study the correlation coefficient has been found to be low, where $r=0.22$. It is concluded that in present study the solar wind southward turning of Bz has significant growth mainly, before the main phase of geomagnetic storm, not during the main phase, which are tested here. Absence of strong correlation between $\mathrm{Bz}$ and Dst during the main phase does not mean that solar wind southward magnetic field component $\mathrm{Bz}$ is not a geo effective parameter.

Investigation shows that strong geomagnetic storms are not necessarily associated with high values of solar wind density. This means that the intensity of a geomagnetic storm is not determined by the variation of plasma density. The weak correlation ( -0.24$)$ between Dst and density is expected (see Figure 3 ).

We are aware that geomagnetic activity is the result of a complex solar wind-magnetosphere interaction process and that solar wind velocity is one of the possible contributing factors. Since the compression and depression in the magnetosphere are supposed to be dependent on the bow shock, proportionality between solar wind velocities and Dst is expected. Although we have found the correlation $(r=-0.39)$ was unexpectedly weak in see Figure 4. The scatter is small and most points lie near a fixed area of speed range and saturated on average value, with a wide range of velocities varying between 400 and $900 \mathrm{~km} / \mathrm{s}$. The more intense geomagnetic storms are not associated with large values of solar wind velocities.

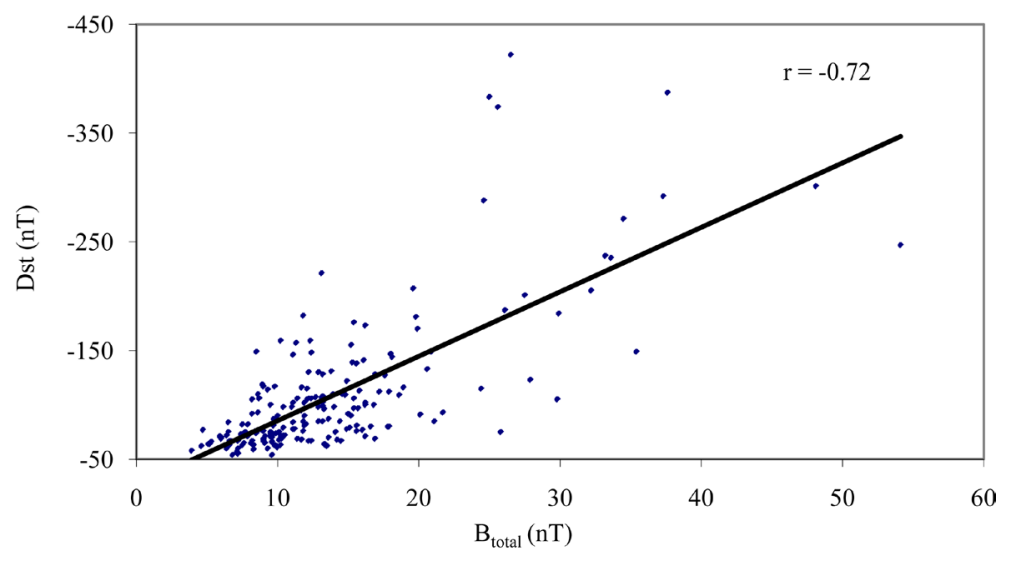

Figure 1. Presents the interplanetary magnetic field $\mathrm{B}_{\text {total }}$ versus Dst minimum. 


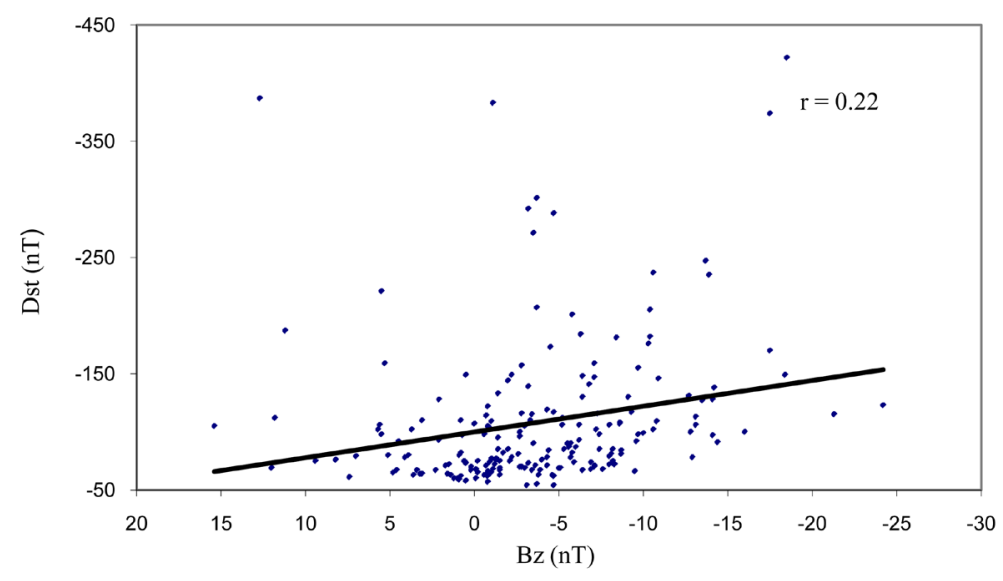

Figure 2. Presents the $\mathrm{Bz}$ (southward component of IMF) versus Dst minimum.

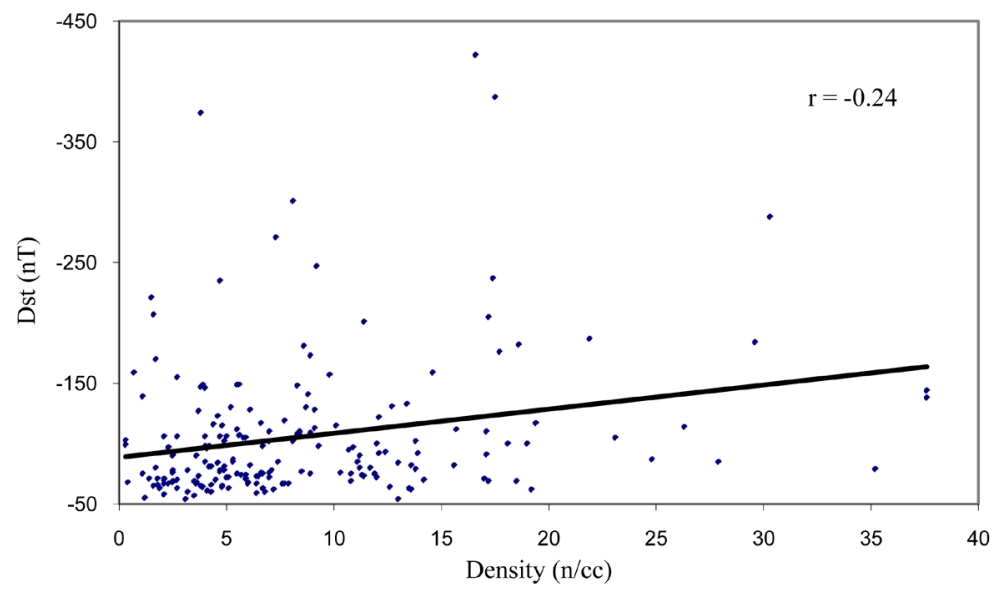

Figure 3. Presents the proton density versus Dst minimum.

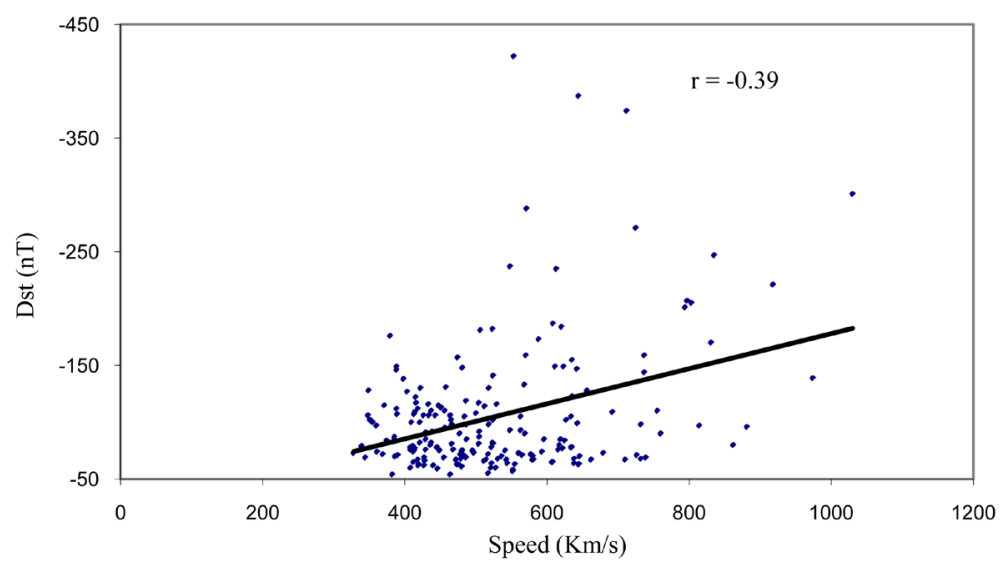

Figure 4. Presents the solar wind speed V versus Dst minimum.

Figure 5 shows the Temperature versus the maximum Dst at the time of Dst peak. For selected event the value of solar wind temperature has large range but most of event occurred when temperature value less than 200,000 K, which show in Figure 5. But weak correlation found between plasma temperature and Dst. It is clearly shows in scattered Figure 5, intense and severe storms produced at the low plasma temperature.

The plasma beta defined as the ratio between the thermal and magnetic energy of the plasma is of the order of 0.0001 - 0.1. In Figure 6 shows the plasma beta versus Dst at the instant of Dst minimum. It is clear from the 


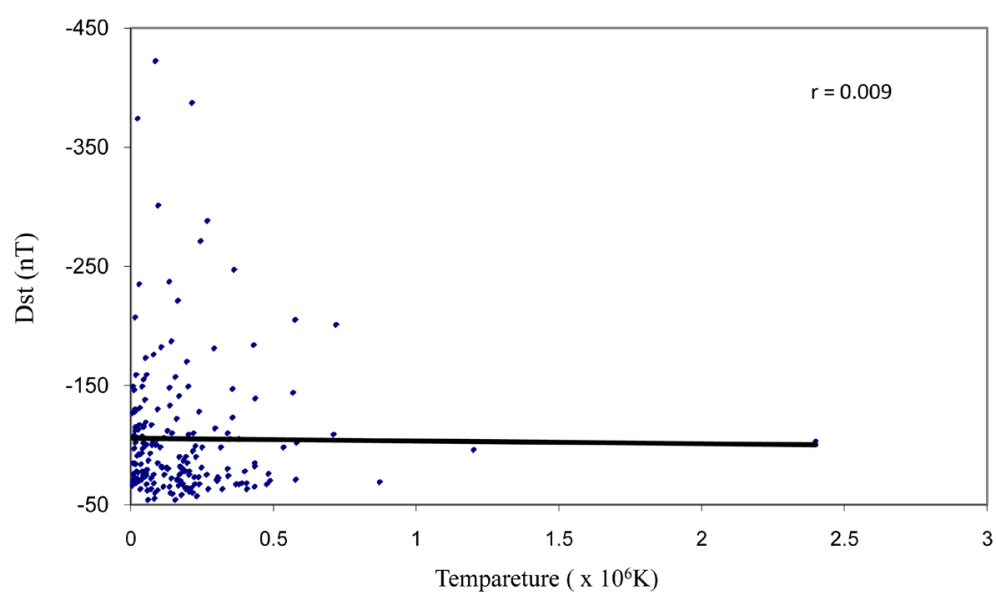

Figure 5. Presents plasma temperature versus Dst minimum.

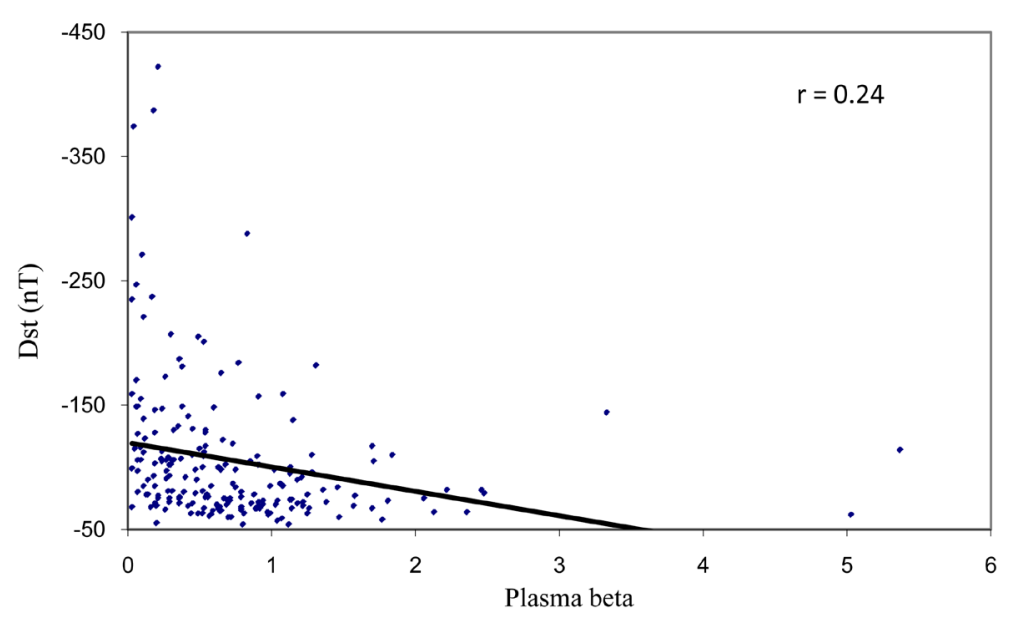

Figure 6. Presents plasma beta versus Dst minimum.

Figure 6, no definite relation found between Dst and plasma beta, correlation occurred very low only 0.24 but it is very clear from figure the Dst value is high at low plasma beta.

To ascertain the dependence of geomagnetic indices on the parameters of solar wind and interplanetary medium during solar cycle-23 events several combination were tried. The one promising candidate is found in the form of solar wind pressure. Figure 7 shows this dependence on Dst. In Figure 7 best fit lines is shown, which indicate that linear relationship between Dst and solar wind pressure (nPa).

\section{Conclusion}

In previous studies, many researchers [16]-[18] have noted that the interplanetary disturbances directed towards the Earth are geo-effective and they give at least a storm sudden commencement (SSC). Earlier researcher has investigated the $\mathrm{Bz}$ component of IMF are more geoeffective. In present study a linear correlation between $\mathrm{B}_{\text {total }}$ and Dst can be seen, that is the strength of the geomagnetic storm is strongly dependent on the total magnetic field $\mathrm{B}_{\text {total }}$. The correlation coefficient has been found to be reasonably high $(-0.72)$. According to previous studies the strength of the geomagnetic storm is strongly dependent on the southward component Bz. But in present study the correlation coefficient has been found to be low (0.22). This result may be obvious solar wind southward magnetic field component $\mathrm{Bz}$ has significant growth mainly before the main phase of geomagnetic storm (not during the main phase, tested here). Thus, this study has something special, which needs to be understand. It is not essential the peak of Bz occurred at the time of Dst minimum. This shows time delay between $\mathrm{Bz}$ and Dst peak. It is clear from this study, the negative $\mathrm{Bz}$ are more geoeffective than positive $\mathrm{Bz}$, but some time geomagnetic storms occur due to positive Bz. Most effective parameter for producing the large-scale geomagnetic 


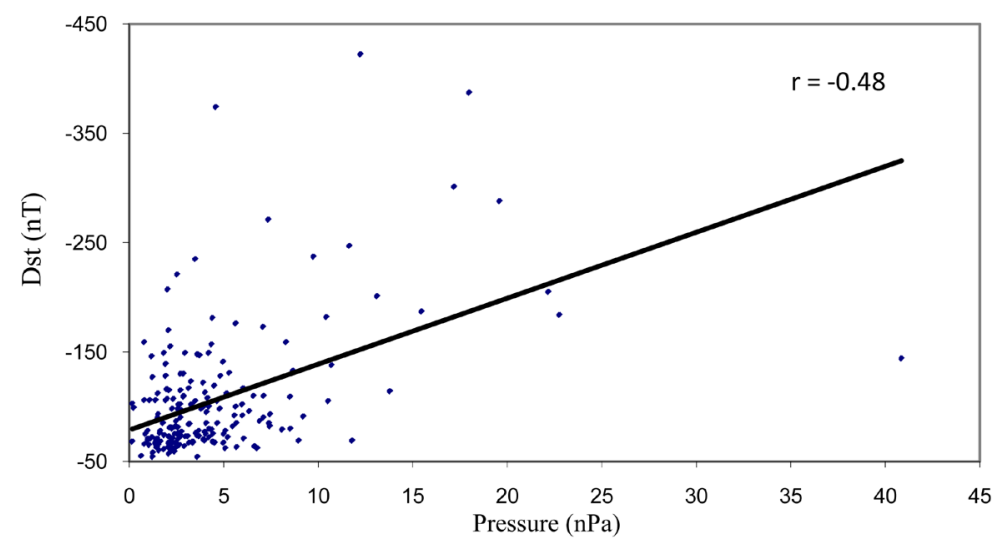

Figure 7. Presents plasma pressure versus Dst minimum.

disturbances is the total interplanetary magnetic field. The present result very clearly indicates that neither Density $\mathrm{N}$ nor temperature is significantly effective in producing geomagnetic disturbance. Rather, it is competing effects of Pressure and V (speed), which are quite effective in producing large-scale geomagnetic disturbances.

\section{Acknowledgements}

Author thanks to Indian Space Research Organization (ISRO) for providing financial support for COSPER meeting during July-2012 at Mysore (India).

\section{References}

[1] Firoz, K.A. (2008) Cosmic Rays and Space Weather. Ph.D. Thesis, Institute of Physics, University of Pavol Jozef Safarik, Slovak Republic.

[2] Rathore, B.S., Kaushik, S.C., Firoz, K.A., Gupta, D.C., Shrivastva, A.K., Parashar, K.K. and Bhadoria, R.M. (2011) A Correlative Study of Geomagnetic Storms Associated with Solar Wind and IMF Features During Solar Cycle-23. International Journal of Applied Physics and Mathematics, 1, 149-154. http://dx.doi.org/10.7763/IJAPM.2011.V1.29

[3] Gonzalez, W.D., Joselyn, J.A., Kamide, Y., Kroehi, H.W., Rostoker, G., Tsurutani, B.T. and Vasylianas, V.M. (1994) What Is a Geomagnetic Storm? Journal of Geophysical Research, 99, 5771. http://dx.doi.org/10.1029/93JA02867

[4] Echer, E., Alves, M.V. and Gonzalez, W.D. (2004), Geoeffectiveness of Interplanetary Shocks during Solar Minimum (1995-1996) and Solar Maximum (2000), Solar Physics, 221, 361-380.

[5] Gonzalez, W.D. and Tsurutani, B.T. (1987) Dual-Peak Solar Cycle Distribution of Intense Geomagnetic Storms. Planetary and Space Science, 38, 181. http://dx.doi.org/10.1016/0032-0633(90)90082-2

[6] Gopalswamy, N., Akiyama, S., Yashiro, S., Michalek, G. and Lepping, R.P. (2008) Solar Sources and Geospace Consequences of Interplanetary Magnetic Clouds Observed during Solar Cycle 23. Journal of Atmospheric and SolarTerrestrial Physics, 70, 245.

[7] Joshi, N.C., Bankoti, N.S., Pande, S., Pande, B. and Pandey, K. (2011) Relationship between Interplanetary Field/ Plasma Parameters with Geomagnetic Indices and Their Behavior during Intense Geomagnetic Storms. New Astronomy, 16, 366-385. http://dx.doi.org/10.1016/j.newast.2011.01.004

[8] Rathore, B.S., Kaushik, S.C. Bhadoria, R.M., Parashar, K.K. and Gupta, D.C. (2012) Sunspots and Geomagnetic Storms during Solar Cycle-23. Indian Journal of Physics, 86, 563-567. http://dx.doi.org/10.1007/s12648-012-0106-2

[9] Sugiura, M. (1964) Annals of the International Geophysical Year, Vol. 35. Pergamon Press, Oxford. 945.

[10] Russell, C.T., McPherron, R.L. and Burton, R.K. (1974) On the Cause of Magnetic Storms. Journal of Geophysical Research, 79, 1105-1109. http://dx.doi.org/10.1029/JA079i007p01105

[11] Burton, R.K., McPherron, R.L. and Russell, C.T. (1975) An Empirical Relationship between Interplanetary Conditions and Dst. Journal of Geophysical Research, 80, 4204-4214. http://dx.doi.org/10.1029/JA080i031p04204

[12] Gonzalez, W.D., Tsurutani, B.T. and de Gonzalez, A.L.C. (1999) Interplanetary Origin of Geomagnetic Storms. Space Science Reviews, 88, 529-562. http://dx.doi.org/10.1023/A:1005160129098

[13] O’Brien, T.P. and McPherron, R.L. (2000) An Empirical Phase Space Analysis of Ring Current Dynamics: Solar Wind Control of Injection and Decay. Journal of Geophysical Research, 105, 7707-7719. 
http://dx.doi.org/10.1029/1998JA000437

[14] Huttunen, K.E.J., Koskinen, H.E.J. and Schwenn, R. (2002) Variability of Magnetospheric Storms Driven by Different Solar Wind Perturbations. Journal of Geophysical Research, 107, JA900171.

[15] Rusanov, A.A. and Pertrukovich, A.A. (2004) Influence Field Fluctuations. Kosmicheskie Issledovania, 42, $368-375$.

[16] Dwivedi, V.C., Tiwari, D.P. and Agrawal, S.P. (2009) Study of the Long-Term Variability of Interplanetary Plasma and Fields as a Link for Solar-Terrestrial Relationships. Journal of Geophysical Research, 114.

[17] Rangarajan, G.K. and Barreto, L.M. (2000) Long Term Variability in Solar Wind Velocity and IMF Intensity and the Relationship between Solar Wind Parameters \& Geomagnetic Activity. Earth, Planets and Space, 52, 121-132. http://dx.doi.org/10.1186/BF03351620

[18] Kane, R.P. (2005) How Good Is the Relationship of Solar and Interplanetary Plasma Parameters with Geomagnetic storm? Journal of Geophysical Research, 110, A02213. 
Scientific Research Publishing (SCIRP) is one of the largest Open Access journal publishers. It is currently publishing more than 200 open access, online, peer-reviewed journals covering a wide range of academic disciplines. SCIRP serves the worldwide academic communities and contributes to the progress and application of science with its publication.

Other selected journals from SCIRP are listed as below. Submit your manuscript to us via either submit@scirp.org or Online Submission Portal.
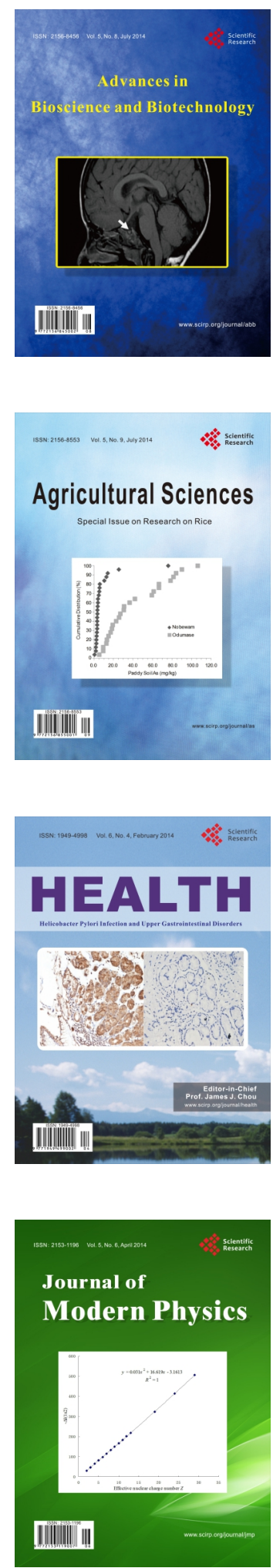
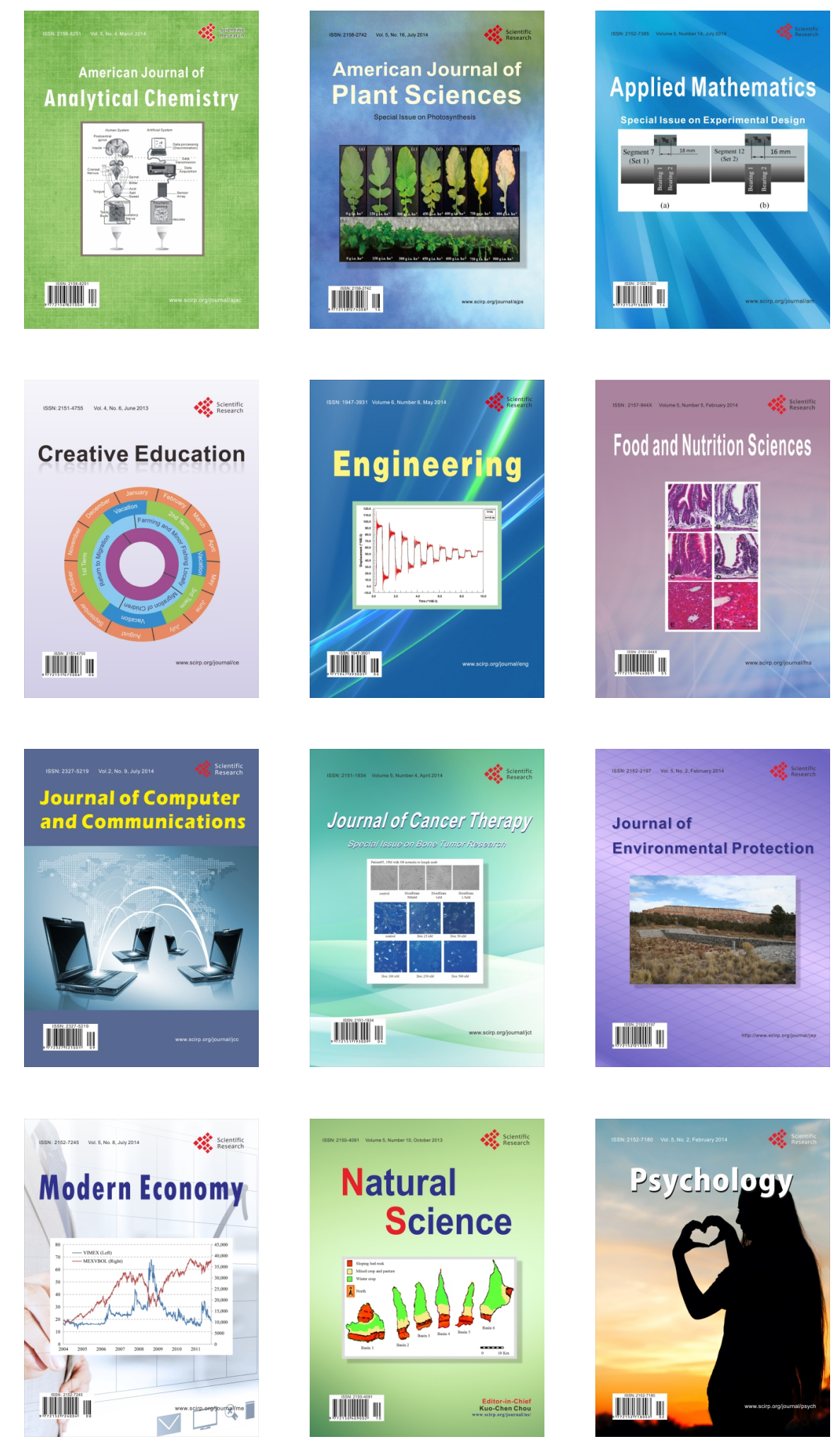